\title{
Risky Lifestyle Behaviors among Gastric Cancer Survivors Compared with Matched Non-cancer Controls: Results from Baseline Result of Community Based Cohort Study
}

\author{
Minkyung Kim, PharmD, MS \\ Kui Son Choi, $\mathrm{PhD}^{1,2}$ \\ Mina Suh, MD, PhD² \\ Jae Kwan Jun, MD, PhD',2 \\ Kumban Walter Chuck, MPH \\ Boyoung Park, MD, PhD',2
}

${ }^{1}$ Department of Cancer Control and Population Health, National Cancer Center Graduate School of Cancer Science and Policy, Goyang, ${ }^{2}$ National Cancer Control Institute, National Cancer Center, Goyang, Korea

\begin{abstract}
Purpose
This study investigated the prevalence of smoking, drinking, and physical inactivity and the associated factors of these behaviors in gastric cancer survivors.

\section{Materials and Methods}

The baseline data from the nationwide cohort study was used. Four hundred thirty-seven gastric cancer survivors who survived $\geq 2$ years from diagnosis and reported completion of treatment were matched with 4,370 controls according to age, sex, education, and household income.

\section{Results}

The prevalence rates of current smoking and drinking among gastric cancer survivors were $8.7 \%$ and $38.3 \%$, which were significantly lower than those among matched controls ( $p \leq 0.001)$, but the prevalence rates of physical inactivity were not significantly different (55.8\% vs. $59.9 \%, p=0.248$ ). In gastric cancer survivors, $\geq 5$ years since cancer diagnosis and current drinking contributed to more current smoking; otherwise, age increment and female were associated with lower current smoking. Those with household income $\geq \$ 2,000$ and current smokers were more likely to drink and female showed less drinking. Female, currently employed state, and self-rated health status were associated with more physical inactivity.

\section{Conclusion}

Although gastric cancer survivors showed better health behaviors than controls, suggesting that the diagnosis of cancer may motivate individuals towards healthy behaviors, their current prevalence rates of smoking and drinking were still high, and more than $50 \%$ of them were physically inactive. Tailored interventions to improve their health behaviors considering associated factors for the gastric cancer survivors are necessary.
\end{abstract}

\author{
Correspondence: Boyoung Park, MD, PhD \\ Department of Cancer Control and Population \\ Health, National Cancer Center Graduate School \\ of Cancer Science and Policy, 323 Ilsan-ro, \\ Ilsandong-gu, Goyang 10408, Korea \\ Tel: 82-31-920-2914 \\ Fax: 82-31-920-2759 \\ E-mail: hayejine@ncc.re.kr \\ Received March 12, 2017 \\ Accepted July 11, 2017 \\ Published Online July 24, 2017
}

\author{
Key words \\ Stomach neoplasms, Survivors, Health behavior, \\ Cross-sectional studies
}

\section{Introduction}

The number of cancer survivors worldwide has increased as the survival rate after a cancer diagnosis has improved [1]. In 2012, there were 32.6 million cancer survivors after being diagnosed with cancer for 5 years or more worldwide [1,2]. Cancer survivors are living in a burden of cancer [3]; thus, prevention of the possible secondary effects from cancer and related cancer burden should be considered. Several studies noted that health behaviors such as smoking, drinking, and physical inactivity patterns of cancer survivors are intimately linked with increased risk of morbidity [3,4]; thus, identification and modification of health behaviors in cancer survivors should be considered.

The incidence of gastric cancer in Eastern Asia is comparatively higher than that of in Western countries [5], which is ranked second for the cancer incidence and third for the 5 -year prevalence among other cancer types [1]. It is reported that not only the incidence but also the prevalence of gastric 
cancer in Eastern Asia are one of the highest in the world $[1,5,6]$. These findings indicate that health behavior and associated sociodemographic factors with them in gastric cancer survivors are one of the most important issues in cancer survivor management. Although researchers' attention has been devoted to evaluating health behavior patterns of cancer survivors in Western countries [7-9], the majority of findings were not focused on gastric cancer which is relatively uncommon in these regions [1].

Thus, this study investigated the prevalence of smoking, alcohol drinking, and physical inactivity in gastric cancer survivors compared to non-cancer controls and the associated sociodemographic factors with aforementioned healthrelated characteristics, using population based data in Korea where gastric cancer incidence is one of the highest worldwide [5].

\section{Materials and Methods}

\section{Study population}

Baseline data from Health Examinees (HEXA) study, which is a part of the nationwide cohort study, the Korea Genome Epidemiology Study (KoGES) was applied. A total of 167,169 subjects aged 40-79, who gave written consent forms, were collected from 38 community-based health examination centers located in eight regions in Korea by face to face interviews using an established questionnaire between 2004 and 2013. The composition of the questionnaire included socio-demographic factors, past medical history, family history, smoking and alcohol drinking, physical activity, dietary habits, socio-psychological factors, and reproductive factors. In addition, their physical examination and clinical lab tests results were collected. Details of the HEXA study are described elsewhere [10].

\section{Case and control selection}

There were 758 self-declared participants who had been diagnosed with gastric cancer on the questionnaire. Considering that the acute survival takes place after the first year treatment [11], we selected gastric cancer patients who survived $\geq 2$ years from the diagnosis and reported that they completed treatment. Thus, 437 gastric cancer survivors $(232$ male and 205 female survivors) were included. Their mean age was 58 years old, including $36.8 \%$ of 2 to 5 years since diagnosis and $62.0 \%$ of $\geq 6$ years since diagnosis. The participants without history of any types of cancer were randomly selected 1:10 ratio by frequency matching with gastric cancer survivors according to sex, 5-year age group, education level $(<$ high school or $\geq$ high school in male and $<$ middle school, middle school, or $\geq$ high school in female), and household income $(<\$ 2,000, \$ 2,000$ to $<\$ 4,000$, or $\geq \$ 4,000$ per month in male and $<\$ 1,500, \$ 1,500$ to $<\$ 3,000$, or $\geq \$ 3,000$ in female considering different education and income level according to sex). Thus, 437 gastric cancer survivors and 4,370 matched controls were included in the study.

\section{Variables}

The outcomes of this study included current smoking, current drinking, and physical activity status in gastric cancer survivors, compared with matched control group. Current smokers were defined as follows: those who reported $\geq 100$ life time cigarettes and smoked at the time of surveying. In addition, those who had smoked $\geq 100$ lifetime cigarettes but did not smoke at the time of the survey were classified as past smokers. Those who reported to have current drinking habit were defined as current drinkers and those who reported to have past drinking habit but quit drinking were defined as past drinkers. The drinking habit included any types of alcohol consumption. Those who reported performing moderate to vigorous activity $<150$ minutes per week were classified as physically inactive and those who reported performing moderate to vigorous activity $\geq 150$ minutes per week were defined as physically active following previous guidelines for cancer survivors [12].

To identify the associated factors with these health behaviors in gastric cancer survivors, age, sex, marital status, education attainment, household income, employment state, self-rated health status, family history of cancer, years since diagnosis, body mass index (BMI), and comorbid chronic disease were considered. The comorbid chronic disease was defined as any of the following diseases: hypertension, diabetes, dyslipidemia, stroke, transient ischemic attacks, angina or myocardial infarction, colon polyp, fatty liver, chronic liver disease or liver cirrhosis, gallbladder stone or cholecystitis, thyroid disease, arthritis, and osteoporosis.

\section{Statistical analysis}

The distribution of health behaviors including current smoking and drinking, physical inactivity, and baseline sociodemographic characteristics between gastric cancer survivors and matched controls were compared by a chi-square test. Among the gastric cancer survivors, the associations the associated factors with each risky health behavior-current smoking, current drinking, current physical activity statuswere assessed using multivariate logistic regression and the results were presented as odds ratio (OR) and 95\% confidence intervals (CIs). Statistical results were analyzed using 
Table 1. Comparison of characteristics between gastric cancer survivors and matched non-cancer controls

\begin{tabular}{|c|c|c|c|}
\hline Characteristic $^{\text {a) }}$ & Survivors $(n=437)$ & Non-cancer controls $(n=4,370)$ & p-value ${ }^{\text {) }}$ \\
\hline Age, mean $(95 \% \mathrm{CI})$ & $58.6(57.9-59.4)$ & $58.5(58.2-58.7)$ & 0.650 \\
\hline \multicolumn{4}{|l|}{ Age (yr) } \\
\hline$<50$ & $64(14.7)$ & $640(14.7)$ & 1.000 \\
\hline $50-54$ & $75(17.2)$ & $750(17.2)$ & \\
\hline $55-59$ & $75(17.2)$ & $750(17.2)$ & \\
\hline$\geq 60$ & $223(51.0)$ & $2,230(51.0)$ & \\
\hline \multicolumn{4}{|l|}{ Sex } \\
\hline Male & $232(53.1)$ & $2,320(53.1)$ & 1.000 \\
\hline Female & $205(46.9)$ & $2,050(47.0)$ & \\
\hline \multicolumn{4}{|l|}{ Marital status } \\
\hline Married, cohabitation & 399 (91.7) & $3,856(88.7)$ & 0.054 \\
\hline Divorced, widow, unmarried & $36(8.3)$ & $492(11.3)$ & \\
\hline \multicolumn{4}{|l|}{ Educational attainment } \\
\hline$\leq$ High school & $323(74.8)$ & $3,178(73.5)$ & 0.562 \\
\hline > College & $109(25.2)$ & $1,147(26.5)$ & \\
\hline \multicolumn{4}{|l|}{ Household income } \\
\hline$<\$ 2,000$ & $186(48.1)$ & $1,815(46.8)$ & 0.629 \\
\hline$\geq \$ 2,000$ & $201(52.0)$ & $2,065(53.2)$ & \\
\hline \multicolumn{4}{|l|}{ Employment state } \\
\hline Currently unemployed & $230(53.1)$ & $2,037(47.6)$ & 0.029 \\
\hline Currently employed & $203(46.9)$ & $2,242(52.4)$ & \\
\hline \multicolumn{4}{|l|}{ Self-rated health status } \\
\hline Healthy & $157(36.3)$ & $1,797(41.5)$ & 0.034 \\
\hline Normal-unhealthy & $276(63.7)$ & $2,532(58.5)$ & \\
\hline \multicolumn{4}{|l|}{ Co-morbid chronic disease ${ }^{c}$} \\
\hline Yes & $226(51.7)$ & $2,308(52.8)$ & 0.661 \\
\hline No & $211(48.3)$ & $2,062(47.2)$ & \\
\hline \multicolumn{4}{|l|}{ Family history of cancer (any type) } \\
\hline Yes & $178(41.1)$ & $1,079(24.8)$ & $<0.001$ \\
\hline No & $255(58.9)$ & $3,275(75.2)$ & \\
\hline \multicolumn{4}{|l|}{ Years since diagnosis } \\
\hline $2-5$ & $161(36.8)$ & - & $<0.001$ \\
\hline$\geq 6$ & $271(62.0)$ & - & \\
\hline \multicolumn{4}{|l|}{ Current smoking status } \\
\hline Yes & $38(8.7)$ & $668(15.4)$ & 0.001 \\
\hline No & $397(91.3)$ & $3,685(84.7)$ & \\
\hline \multicolumn{4}{|l|}{ Current drinking status } \\
\hline Yes & $166(38.3)$ & $2,162(49.7)$ & $<0.001$ \\
\hline No & $267(61.7)$ & $2,192(50.3)$ & \\
\hline \multicolumn{4}{|l|}{ Physical activity } \\
\hline$<150 \mathrm{~min} / \mathrm{wk}$ & $242(55.8)$ & 2,521 (59.9) & 0.095 \\
\hline$\geq 150 \mathrm{~min} / \mathrm{wk}$ & $192(44.2)$ & $1,688(40.1)$ & \\
\hline
\end{tabular}

(Continued to the next page) 
Table 1. Continued

\begin{tabular}{|c|c|c|c|}
\hline Characteristic $^{\text {a) }}$ & Survivors (n=437) & Non-cancer controls $(n=4,370)$ & p-value ${ }^{b)}$ \\
\hline \multicolumn{4}{|l|}{ BMI } \\
\hline$<23$ & $278(63.6)$ & $1,535(35.2)$ & $<0.001$ \\
\hline$\geq 23$ & $159(36.4)$ & $2,826(64.8)$ & \\
\hline
\end{tabular}

Values are presented as number (\%) unless otherwise indicated. CI, confidence intervals; BMI, body mass index. ${ }^{\text {a) Missing }}$ values (No. of survivors, No. of non-cancer controls): marital status $(2,22)$, educational attainment $(5,45)$, household income $(50,490)$, employment state $(4,91)$, self-rated health status $(4,41)$, family history of cancer $(4,16)$, current smoking status $(2$, 17), current drinking status $(4,16)$, physical activity $(3,161)$, BMI $(0,9)$, b)Chi-square test or $t$ test results, ${ }^{c}$ Any of following diseases were classified as chronic disease: hypertension, diabetes, dyslipidemia, stroke, transient ischemic attacks, angina or myocardiac infarction, colon polyp, fatty liver, chronic liver disease or liver cirrhosis, gallbladder stone or cholecystitis, thyroid disease, arthritis, and osteoporosis.

SAS ver. 9.3 software (SAS Institute Inc., Cary, NC).

\section{Ethical statement}

The Institutional Review Board of the National Cancer Center approved this study protocol, which was in compliance with the Declaration of Helsinki (IRB No. NCC20140098). All participants provided their written informed consent.

\section{Results}

The comparison of baseline sociodemographic characteristics between 437 gastric cancer survivors and matched 4,370 controls are presented in Table 1. The distributions of matched variables (sex, age, educational attainment, and household income) and the proportion of subjects with comorbidity were similar between the two groups. However, current employment state was lower in gastric cancer survivors and self-rated health status, family history of cancer, and BMI were significantly different between the two groups $(\mathrm{p}<0.05)$.

The prevalence of current smoking and current drinking in gastric cancer survivors were $8.7 \%$ and $38.3 \%$, respectively, which were significantly lower than matched cancer free controls $(15.4 \%$ and $49.7 \%$, respectively; $\mathrm{p}=0.001$ and $\mathrm{p}<0.001)$; otherwise the prevalence of physical inactivity was not significantly different in two groups $(55.8 \%$ and $59.9 \% ; \mathrm{p}=0.095$ ) (Fig. 1A). The differences in smoking and drinking status were significant in male $(\mathrm{p}<0.001)$ (Fig. 1B); however, in female, we did not find significant differences in these three health behaviors (Fig. 1C). Among the non-current smokers and drinkers, the proportion of past smokers and drinkers were high in gastric cancer survivors, especially in male (Fig. 1B).

The relationship between considered sociodemographic factors and health behaviors in gastric cancer survivors is described in Table 2. One-year increment of age and female presented less current smoking status (OR, 0.93; 95\% CI, 0.88 to 0.99 and OR, 0.16 ; $95 \%$ CI, 0.05 to 0.46 ). On the other hand, more than 5 years since gastric cancer diagnosis and current drinking contribute to more current smoking (OR, 3.17; 95\% CI, 1.27 to 7.90 and OR, 2.48; 95\% CI, 1.13 to 5.45). Female was less likely to engage in current drinking (OR, 0.23; 95\% CI, 0.14 to 0.40$)$, whereas household income greater and equal to $\$ 2,000$ and current smoking status are more likely to be associated with current drinking (OR, 1.74; 95\% CI, 1.03 to 2.94 and OR, 2.43; 95\% CI, 1.11 to 5.33). Female, currently employed state, and normal and unhealthy self-rated health status were associated with more physical inactivity (OR, 2.12; $95 \% \mathrm{CI}, 1.26$ to 3.57 and OR, $1.97 ; 95 \% \mathrm{CI}, 1.24$ to 3.12 and $\mathrm{OR}, 1.57 ; 95 \% \mathrm{CI}, 1.02$ to 2.43 ), whereas having co-morbid chronic diseases other than gastric cancer were associated with less physical inactivity (OR, $0.65 ; 95 \% \mathrm{CI}, 0.42$ to 0.99 ).

\section{Discussion}

In this study, health behaviors of gastric cancer survivors and cancer-free controls were compared and the associated factors with current unhealthy behaviors including smoking, and drinking, and physical inactivity in gastric cancer survivors were evaluated. From our findings, gastric cancer survivors were currently engaged with a healthier lifestyle in smoking and drinking compared with the cancer free population-91.3\% did not smoke and $61.7 \%$ did not drink. However, the prevalence of physical inactivity was higher $(55.8 \%)$ 

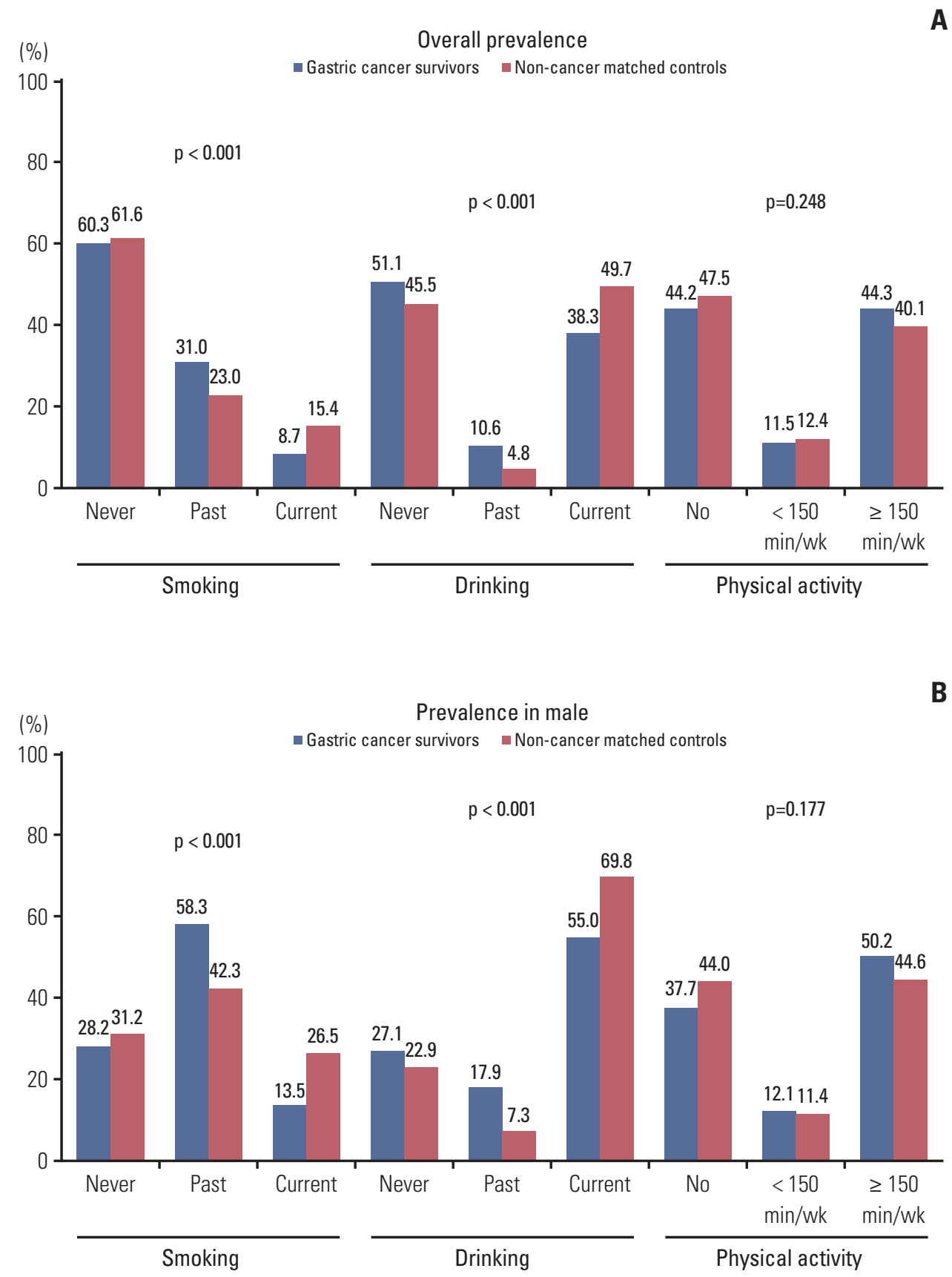

Fig. 1. Prevalence of smoking, drinking, and physical activity status in gastric cancer survivors compared with non-cancer matched controls. (A) Prevalence in both. (B) Prevalence in male. (Continued to the next page)

and it was not better than cancer-free controls. The prevalence of these health behaviors in gastric cancer survivors from this nationwide study was similar to that from a single survivorship center [13]. Regarding smoking, our results were similar to the American Cancer Society's study results: $91.6 \%$ of cancer survivors adhered to the smoking recommendation [14]. Although some previous studies conducted in Western regions showed higher or similar prevalence of current smoking and drinking in cancer survivors, compared with the general population $[15,16]$, studies were done in Canada [17] and Korea [18,19] supported good health behaviors among cancer survivors in smoking and drinking.

Maintaining recommended amounts of physical activity is one of the most important factors for cancer survivors to 


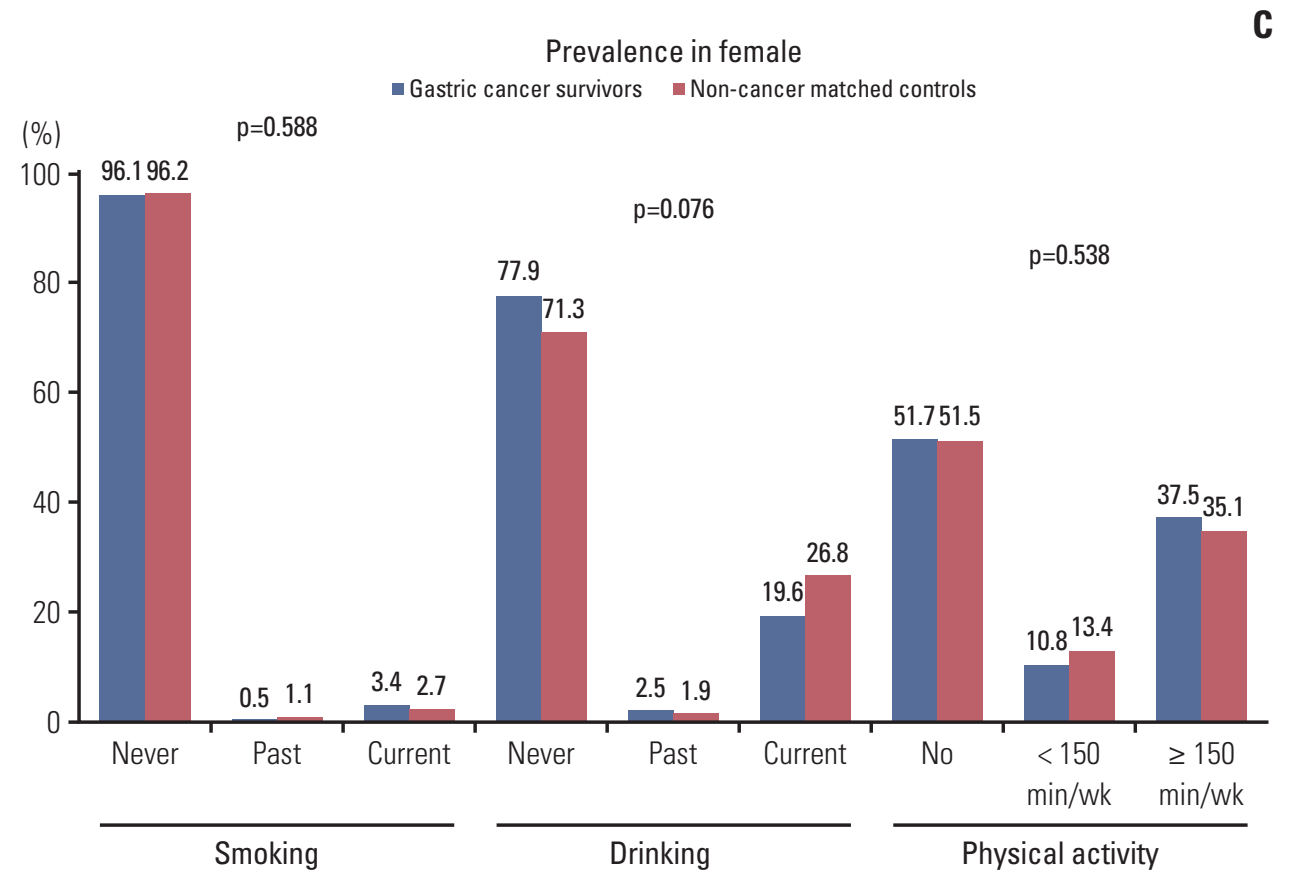

Fig. 1. (Continued from the previous page) (C) Prevalence in female.

decrease mortality $[9,20,21]$. A previous study done in Western populations pointed out that cancer survivors' physical activity status is similar to controls [15]. Our study results were comparable to the results of previous Korean studies, in which the proportion of cancer survivors engaging in physical activity from $26.0 \%$ to $50.7 \%$ [18,19]. Thus, the importance of physical activity in cancer survivors should be reiterated and educated in the care of gastric cancer survivors.

In our findings, it was shown that the health behaviors in female survivors were not different from matched female controls; on the other hand, male gastric cancer survivors showed better health behaviors than controls. Considering the higher proportion of past smoking and drinking in male gastric cancer survivors compared with matched controls (Fig. 1B), gastric cancer survivors, especially male survivors may be more willing to adopt healthy lifestyles such as quitting smoking or drinking. When we compared the health behaviors of gastric cancer survivors by sex, female gastric cancer survivors were engaged with less smoking and drinking but more physical inactive than male gastric cancer survivors. The result was consistent with existing studies which reported that male cancer survivors were associated with more alcohol consumption [22] and current smoking [18], and the multivariate logistic regression analysis in our study also confirmed the association between gender and health behaviors in gastric cancer survivors.
The relationship between socioeconomic status and health behaviors is well known in the general population [6]. However, the existing literature on household income conflicts with the result that the lower household income of cancer survivors affects cancer survivors' health status negatively (including general and mental health) [23], whereas the young adult childhood cancer survivors with higher income were prone to binge drinking [22]. Our results showed the increased tendency of alcohol consumption in gastric cancer survivors with higher household income, which was comparable with the previous study done in Korea [18]. A similar pattern was also observed in the general population in China; it might be due to higher income giving more opportunities engage with social networking in not only the general population [24] but also survivors [18,22].

Physical inactivity was influenced by current employment, self-rate health, and co-morbid chronic disease status $[15,16,18,21,25]$. A previous result offers a good example of long working hours associated with no leisure time for physical activity [26], and it might be a reason for reduced physical activity in gastric cancer survivors in employment status. Cancer survivors whose self-rated health status were marked as normal and unhealthy were engaged in physical inactivity, consistent with previous studies in cancer survivors [27] or the general population [28,29]. Although previous studies did not observe an association between physical activity and chronic disease, surprisingly, gastric cancer survivors who 


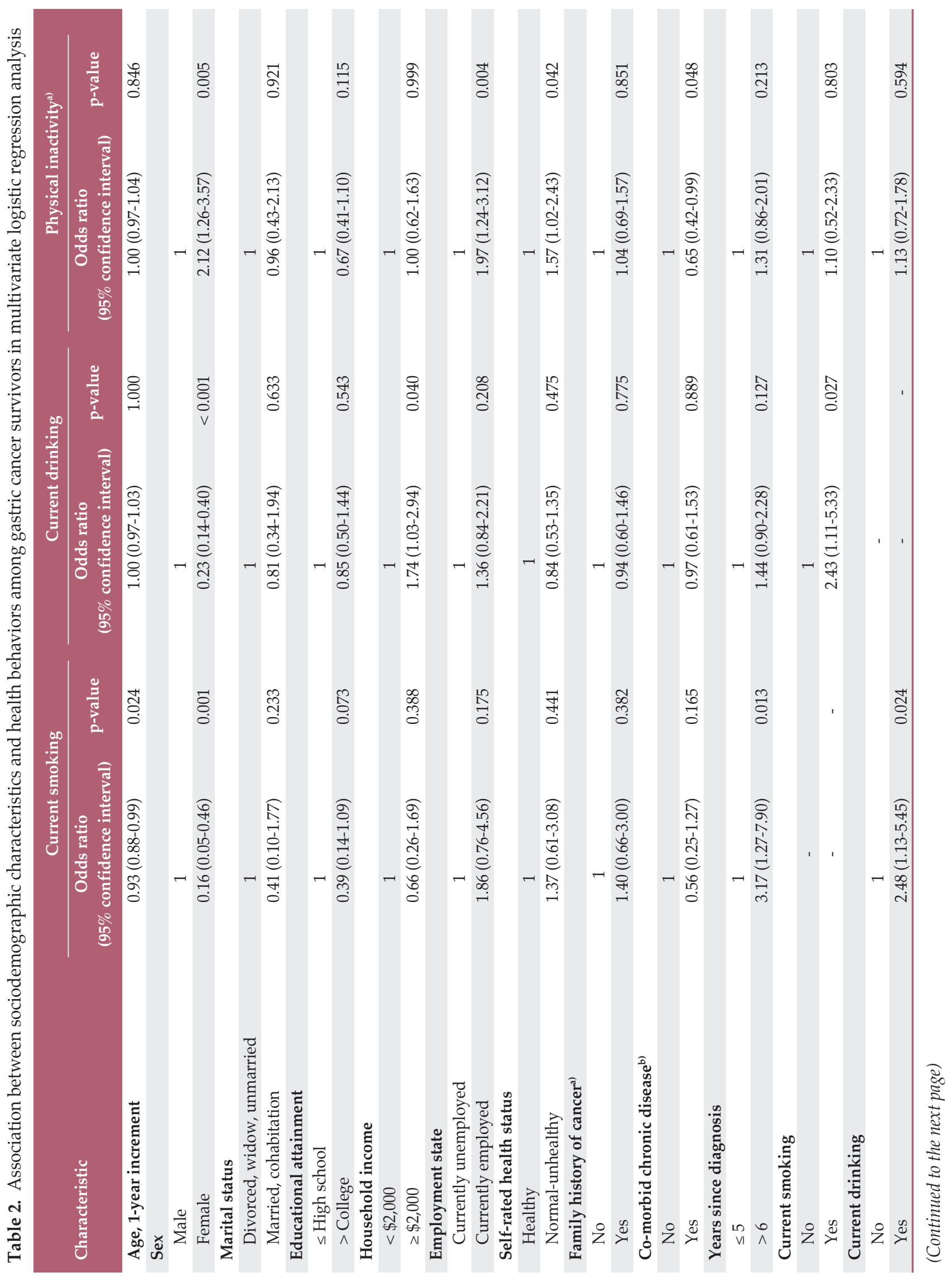




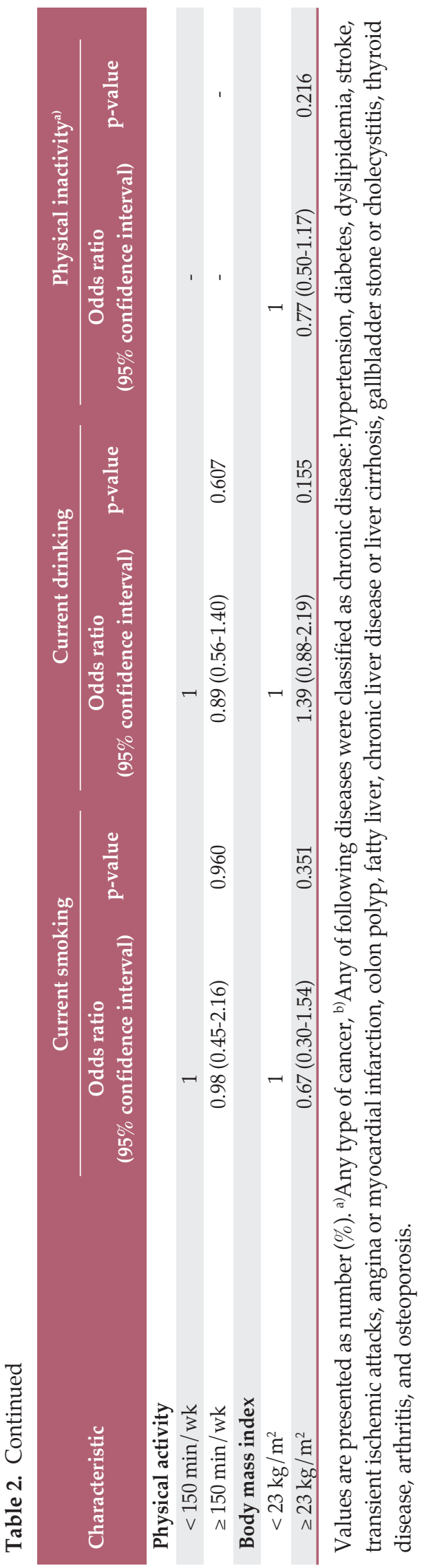

had comorbid conditions were engaged in more physical activity in our study. In Korea, the management of cancer survivors is mostly conducted by cancer specialists $[30,31]$ and the modification of health behaviors through rehabilitation programs has not received much attention in cancer care. In addition, cancer specialists thought they were not well resourced for these programs in Korea [32,33]. However, gastric cancer survivors with chronic diseases may have more opportunity to visit the physicians who managed their chronic diseases to be educated about the importance of exercise. The modification of unhealthy behaviors, especially exercise has been considered to be an important part of the management of chronic diseases [34]. Thus, it is necessary that oncologists stress the importance of physical activity to cancer survivors as it was done in individuals with chronic diseases. There is no doubt of the interrelation among health behavior risk factors in cancer patient; the interdependence between smoking and drinking among cancer survivors were observed in our result as well as in other studies $[18,22]$. One more noticeable this was that gastric cancer survivors diagnosed $\geq 6$ years smoked three times more, suggesting that efforts for long-term lifestyle modification is necessary for gastric cancer survivors.

Despite these findings, there remain some limitations in this study. First, the HEXA data was collected from 38 health examination centers and included individuals over 40 years of age; thus, the individuals in the study might have different social, economic, and health status compared to general population, and our results may not represent whole gastric cancer survivors. Especially, it should be noted that the HEXA data includes individuals over 40 years of age. In addition, survival bias should be considered because only people who survived at the time of the study were included. The information about cancer stage and treatments were not included in this study questionnaire. Thus, the health behavior of cancer survivors in this regard could not be detected. This limitation was observed in several population-based studies on health behaviors in cancer survivors as well $[15,16,35]$. Thus, including the information in questionnaires used in population-based research would be necessary to understand health behaviors in cancer survivors better. Furthermore, as a nature of self-reported data, the range of smoking and alcohol consumption might have been underestimated; on the other hand, the range of physical activity might have been overestimated [16,19]. Although we proposed several sociodemographic factors associated with health behaviors, temporal information and causality could not be addressed due to the cross-sectional approach of this study. To estimate the number of years since diagnosis, age at interview and age at diagnosis of gastric cancer were applied. Because we did not have information about the exact date of cancer diagnosis and interview, there could be some misclassification. 
Notwithstanding these limitations, our study excluded the heterogeneity bias expected in including various cancer sites as previous studies in Korea [18,19]; rather, we focused on gastric cancer which is one of the most commonly prevalent cancers in Asian countries. Gastric cancer survivors who completed treatment and visited health examination centers may more readily return to normal life, and thus, may be primary targets for community-based management for gastric cancer survivors. To increase the comparability of health behaviors, survivors and controls were matched by sex, age, educational level, and income and stratified analysis by sex was performed as well.

To the best of our knowledge, this is the first study to investigate the health behaviors and associated sociodemographic factors in gastric cancer survivors who completed treatment and returned to normal life. Gastric cancer survivors, especially male survivors, presented better health behaviors regarding smoking and drinking compared with controls, but their physical activity level was not better, and the proportion of physical activity below the recommended level was more than $50 \%$. These lifestyle factors are modifiable and improve the prognosis of cancer survivors. However, efforts to induce healthy behavior in gastric cancer survivors are often neglected during cancer treatment and rehabilitation. Several sociodemographic characteristics were associated with unhealthy behaviors among gastric cancer survivors. Identifying gastric cancer survivors vulnerable to unhealthy lifestyles would allow intervention programs to effectively target their limited resources. In addition, further studies are needed to explore why some gastric cancer survivors continue to engage in unhealthy behaviors.

\section{Conflicts of Interest}

Conflict of interest relevant to this article was not reported.

\section{Acknowledgments}

This study was supported by a Grant-in-Aid for Cancer Research and Control from the National Cancer Center of Korea (No. 1710171) to Dr. Boyoung Park. This study used data from the Korean Genome Analysis Project (4845-301), the Korean Genome and Epidemiology Study (4851-302), and the Korea Biobank Project (4851-307, KBP2014-041), which were supported by the Korea Centers for Disease Control and Prevention, Republic of Korea.

\section{References}

1. Ferlay J, Soerjomataram I, Ervik M, Dikshit R, Eser S, Mathers C, et al. GLOBOCAN 2012 v1. 0, cancer incidence and mortality worldwide: IARC CancerBase No. 11 [Internet]. Lyon: International Agency for Research on Cancer; 2013 [cited 2014 Oct 10]. Available from: http://globocan.iarc.fr.

2. Bray F, Ren JS, Masuyer E, Ferlay J. Global estimates of cancer prevalence for 27 sites in the adult population in 2008. Int J Cancer. 2013;132:1133-45.

3. Haugnes HS, Bosl GJ, Boer H, Gietema JA, Brydoy M, Oldenburg J, et al. Long-term and late effects of germ cell testicular cancer treatment and implications for follow-up. J Clin Oncol. 2012;30:3752-63.

4. Leach CR, Weaver KE, Aziz NM, Alfano CM, Bellizzi KM, Kent EE, et al. The complex health profile of long-term cancer survivors: prevalence and predictors of comorbid conditions. J Cancer Surviv. 2015;9:239-51.

5. Rahman R, Asombang AW, Ibdah JA. Characteristics of gastric cancer in Asia. World J Gastroenterol. 2014;20:4483-90.

6. Pampel FC, Krueger PM, Denney JT. Socioeconomic disparities in health behaviors. Annu Rev Sociol. 2010;36:349-70.

7. Newcomb PA, Kampman E, Trentham-Dietz A, Egan KM, Titus LJ, Baron JA, et al. Alcohol consumption before and after breast cancer diagnosis: associations with survival from breast cancer, cardiovascular disease, and other causes. J Clin Oncol.
2013;31:1939-46.

8. Kawahara M, Ushijima S, Kamimori T, Kodama N, Ogawara M, Matsui K, et al. Second primary tumours in more than 2-year disease-free survivors of small-cell lung cancer in Japan: the role of smoking cessation. Br J Cancer. 1998;78:409-12.

9. Schmid D, Leitzmann MF. Association between physical activity and mortality among breast cancer and colorectal cancer survivors: a systematic review and meta-analysis. Ann Oncol. 2014;25:1293-311.

10. Health Examinees Study Group. The Health Examinees (HEXA) study: rationale, study design and baseline characteristics. Asian Pac J Cancer Prev. 2015;16:1591-7.

11. Kunz PL, Gubens M, Fisher GA, Ford JM, Lichtensztajn DY, Clarke CA. Long-term survivors of gastric cancer: a California population-based study. J Clin Oncol. 2012;30:3507-15.

12. Rock CL, Doyle C, Demark-Wahnefried W, Meyerhardt J, Courneya KS, Schwartz AL, et al. Nutrition and physical activity guidelines for cancer survivors. CA Cancer J Clin. 2012;62:243-74.

13. Lee JE, Shin DW, Lee H, Son KY, Kim WJ, Suh YS, et al. Oneyear experience managing a cancer survivorship clinic using a shared-care model for gastric cancer survivors in Korea. J Korean Med Sci. 2016;31:859-65.

14. Blanchard CM, Courneya KS, Stein K; American Cancer Soci- 
ety's SCS-II. Cancer survivors' adherence to lifestyle behavior recommendations and associations with health-related quality of life: results from the American Cancer Society's SCS-II. J Clin Oncol. 2008;26:2198-204.

15. Eakin EG, Youlden DR, Baade PD, Lawler SP, Reeves MM, Heyworth JS, et al. Health behaviors of cancer survivors: data from an Australian population-based survey. Cancer Causes Control. 2007;18:881-94.

16. Bellizzi KM, Rowland JH, Jeffery DD, McNeel T. Health behaviors of cancer survivors: examining opportunities for cancer control intervention. J Clin Oncol. 2005;23:8884-93.

17. Carswell K, Chen Y, Nair RC, Shaw AK, Speechley KN, Barrera $\mathrm{M}$, et al. Smoking and binge drinking among Canadian survivors of childhood and adolescent cancers: a comparative, population-based study. Pediatr Blood Cancer. 2008;51:280-7.

18. Park B, Kong SY, Kim J, Kim Y, Park IH, Jung SY, et al. Health behaviors of cancer survivors in nationwide cross-sectional survey in Korea: higher alcohol drinking, lower smoking, and physical inactivity pattern in survivors with higher household income. Medicine (Baltimore). 2015;94:e1214.

19. Oh MG, Han MA, Park J, Ryu SY, Park CY, Choi SW. Health behaviors of cancer survivors: the Fourth Korea National Health and Nutrition Examination Survey (KNHANES IV, 2007-09). Jpn J Clin Oncol. 2013;43:981-7.

20. Kushi LH, Doyle C, McCullough M, Rock CL, Demark-Wahnefried W, Bandera EV, et al. American Cancer Society Guidelines on nutrition and physical activity for cancer prevention: reducing the risk of cancer with healthy food choices and physical activity. CA Cancer J Clin. 2012;62:30-67.

21. Ballard-Barbash R, Friedenreich CM, Courneya KS, Siddiqi SM, McTiernan A, Alfano CM. Physical activity, biomarkers, and disease outcomes in cancer survivors: a systematic review. J Natl Cancer Inst. 2012;104:815-40.

22. Rebholz CE, Kuehni CE, Strippoli MP, Rueegg CS, Michel G, Hengartner $\mathrm{H}$, et al. Alcohol consumption and binge drinking in young adult childhood cancer survivors. Pediatr Blood Cancer. 2012;58:256-64.

23. Hudson MM, Mertens AC, Yasui Y, Hobbie W, Chen H, Gurney JG, et al. Health status of adult long-term survivors of childhood cancer: a report from the Childhood Cancer Survivor Study. JAMA. 2003;290:1583-92.

24. Li Y, Jiang Y, Zhang M, Yin P, Wu F, Zhao W. Drinking behaviour among men and women in China: the 2007 China Chronic Disease and Risk Factor Surveillance. Addiction. 2011;106:1946-56.

25. Fong DY, Ho JW, Hui BP, Lee AM, Macfarlane DJ, Leung SS, et al. Physical activity for cancer survivors: meta-analysis of randomised controlled trials. BMJ. 2012;344:e70.

26. Artazcoz L, Cortes I, Escriba-Aguir V, Cascant L, Villegas R. Understanding the relationship of long working hours with health status and health-related behaviours. J Epidemiol Community Health. 2009;63:521-7.

27. Lee HY, Kim J, Merighi JR. Physical activity and self-rated health status among older adult cancer survivors: does intensity of activity play a role? Oncol Nurs Forum. 2015;42:614-24.

28. Cimarras-Otal C, Calderon-Larranaga A, Poblador-Plou B, Gonzalez-Rubio F, Gimeno-Feliu LA, Arjol-Serrano JL, et al. Association between physical activity, multimorbidity, selfrated health and functional limitation in the Spanish population. BMC Public Health. 2014;14:1170.

29. Hudon C, Soubhi H, Fortin M. Relationship between multimorbidity and physical activity: secondary analysis from the Quebec health survey. BMC Public Health. 2008;8:304.

30. Shin DW, Kim Y, Baek YJ, Mo HN, Choi JY, Cho J. Oncologists experience with second primary cancer screening: current practices and barriers and potential solutions. Asian Pac J Cancer Prev. 2012;13:671-6.

31. Shin DW, Sunwoo S, Lee J. Management of cancer survivors in Korea. J Korean Med Assoc. 2015;58:216-26.

32. Yang EJ, Chung SH, Jeon JY, Seo KS, Shin HI, Hwang JH, et al. Current practice and barriers in cancer rehabilitation: perspectives of Korean physiatrists. Cancer Res Treat. 2015;47: 370-8.

33. Shin DW, Cho B, Kim SY, Jung JH, Park JH. Management of cancer survivors in clinical and public health perspectives: current status and future challenges in Korea. J Korean Med Sci. 2013;28:651-7.

34. Lorig KR, Ritter P, Stewart AL, Sobel DS, Brown BW Jr,] Bandura A, et al. Chronic disease self-management program: 2-year health status and health care utilization outcomes. Med Care. 2001;39:1217-23.

35. Linsky A, Nyambose J, Battaglia TA. Lifestyle behaviors in Massachusetts adult cancer survivors. J Cancer Surviv. 2011;5: 27-34. 